\title{
Correction to: Change in Obesity Prevalence among New York City Adults: the NYC Health and Nutrition Examination Survey, 2004 and 2013-2014
}

\author{
Pasquale Rummo • Rania Kanchi - Sharon Perlman • \\ Brian Elbel • Chau Trinh-Shevrin • Lorna Thorpe
}

Published online: 20 August 2018

(C) The New York Academy of Medicine 2018

\section{Correction to: J Urban Health}

https://doi.org/10.1007/s11524-018-0288-9

Readers should note the following two typographical errors in this article:

The $1^{\text {st }}$ typo is in the final paragraph of the Introduction, here:

- Original text: We compared changes in obesity prevalence in NYC to corresponding changes at the national level using data from NHANES 2003-2004 and 2011-2012.

- New text: We compared changes in obesity prevalence in NYC to corresponding changes at the national level using data from NHANES 2001-2004 and 2011-2014.

The online version of the original article can be found at https://doi.org/10.1007/s11524-018-0288-9

P. Rummo $(\bowtie) \cdot$ R. Kanchi · B. Elbel • C. Trinh-Shevrin •

L. Thorpe

Department of Population Health, NYU School of Medicine, New York, NY, USA

e-mail: Pasquale.Rummo@nyumc.org

S. Perlman

NYC Department of Health and Mental Hygiene, Division of Epidemiology, Long Island City, NY, USA

B. Elbel

NYU Wagner Graduate School of Public Service, New York, NY, USA
The $2^{\text {nd }}$ typo in the "Study Sample" sub-section of the Methods, here:

- Original text: To obtain national estimates of obesity change, we used data from NHANES 2003-2004 and 2011-2012 [26], with a sample size of 10,927 and 7408 , respectively, after excluding those without valid height and weight measurements.

- New text: To obtain national estimates of obesity change, we used data from NHANES 2001-2004 and 2011-2014 [26], with a sample size of 10,927 and 7408 , respectively, after excluding those without valid height and weight measurements. 